\title{
Applications of Prüfer Transformations in the Theory of Ordinary Differential Equations
}

\author{
GEORGE CHAILOS
}

\begin{abstract}
This article is a review article on the use of Prüfer Transformations techniques in proving classical theorems from the theory of Ordinary Differential Equations. We consider self-adjoint second order linear differential equations of the form

$$
\mathcal{L} x=\left(p(t) x^{\prime}(t)\right)^{\prime}+g(t) x(t)=0, t \in(a, b) .
$$

We use Prüfer transformation techniques (which are a generalization of Poincaré phase-plane analysis) to obtain some of the main theorems of the classical theory of linear differential equations. First we prove theorems from the Oscillation Theory (Sturm Comparison theorem and Disconjugacy theorems). Furthermore we study the asymptotic behavior of the equation $(\star)$ when $t \rightarrow \infty$ and we obtain necessary and sufficient conditions in order to have bounded solutions for $(\star)$. Finally, we consider a certain type of regular Sturm-Liouville eigenvalue problems with boundary conditions and we study their spectrum via Prüfer transformations.
\end{abstract}

\section{INTRODUCTION}

In this review article we will present main theorems related to the study of the solutions of self-adjoint second order linear Differential Equations of the form

$$
\mathcal{L} x=\left(p(t) x^{\prime}(t)\right)^{\prime}+g(t) x(t)=0, t \in(a, b),
$$

where $p(t)>0, p(t)$ is absolutely continuous and $g(t) \in L^{1}(a, b)$ where $a, b$ are elements in the extended real line. For this, we will develop and use the so called "Prüfer transformations" which are (roughly speaking) a generalization of the Poincaré phase plane

2000 Mathematics Subject Classification. 65L05, 34B24, 34L15.

Key words and phrases. Ordinary Differential Equations, Prüfer Transformations, Sturm-Liouville Problems. 
analysis. The "Prüfer transformations" are general polar coordinate representations of the solutions of (1.1). The most common Prüfer transformation is

$$
\left\{\begin{array}{l}
x(t)=r(t) \sin \Theta(t) \\
x^{\prime}(t)=\frac{r(t)}{p(t)} \cos \Theta(t) .
\end{array}\right.
$$

Substituting (1.2) to (1.1) we obtain the Prüfer system

$$
\left\{\begin{array}{l}
r^{\prime}(t)=\left(\frac{1}{p(t)}-g(t)\right) r \sin \Theta \cos \Theta \\
\Theta^{\prime}(t)=\frac{1}{p(t)} \cos ^{2} \Theta+g(t) \sin ^{2} \Theta .
\end{array}\right.
$$

In the second section (Oscillation Theory) we will use the transformed system (1.3) in order to prove main theorems from the Oscillation theory, like the Sturm Comparison Theorem, the Oscillation theorem, and Disconjugacy theorems. In the third section (Bounds of Solutions and Asymptotic Behavior) we will use the Prüfer transformation and the modified Prüfer transformation in order to study the asymptotic behavior of the equation (1.1) when $t \rightarrow \infty$ (without considering that $g(t) \in L^{1}(m)$ ). Moreover we will prove necessary and sufficient conditions in order to have bounded solutions for (1.1). Finally, in the last section (Spectral Theory) we will consider the regular Sturm-Liouville eigenvalue problem with boundary conditions,

$$
\left\{\begin{array}{l}
\left(p(t) x^{\prime}(t)\right)^{\prime}+(\lambda r(t)-q(t)) x(t)=0, \quad t \in[a, b], \lambda \neq 0 \\
A x(a)-B x^{\prime}(a)=0 \\
\Gamma x(b)-\Delta x^{\prime}(b)=0 .
\end{array}\right.
$$

We will use the Prüfer transformation to prove that there is an infinite number of eigenvalues of (1.4) forming a monotone increasing sequence with $\lambda_{n} \rightarrow \infty$, and that the eigenfunctions $\Phi_{n}$ corresponding to the eigenvalues $\lambda_{n}$ have exactly $\mathrm{n}$ zeros in $(\mathrm{a}, \mathrm{b})$. Moreover, we will use the Prüfer transformed system to derive upper and lower bounds for the spectrum of (1.4).

At the end we present a list of references which were used in this article. The reader may refer to them for proofs that are not included in this paper.

I would like to note that I am particularly in debt to Professor D. Hinton for his constant willingness to discuss each step of this paper. 


\section{Oscillation Theory}

In this section we will apply the Prüfer transformation on regular Sturm-Liouville problems in order to prove the Sturm Comparison theorem, the Oscillation theorem and "Disconjugacy" theorems.

Consider the equation of the form

$$
\mathcal{L} x=\left(p(t) x^{\prime}\right)^{\prime}+g(t) x=0, \quad t \in(a, b) .
$$

(Note that the equation $x^{\prime \prime}+f(t) x^{\prime}+h(t) x=0$ can be transformed in the form of (2.1) by multiplying it with $e^{\int_{0}^{t} f(s) d s}$.) We assume that $p(t)>0$ with $p$ absolutely continuous and $g \in L^{1}(m)$.

In (2.1) we consider the substitution $y=p(t) x^{\prime}$. From (2.1),

$$
x^{\prime}=\frac{y}{p(t)}, \quad y^{\prime}=-g(t) x .
$$

If we use polar coordinates, $x=r(t) \sin \theta(t), y=r(t) \cos \theta(t)$ on (2.2), and solve for $r^{\prime}, \theta^{\prime}$, then we obtain the Prüfer system

$$
\begin{aligned}
r^{\prime}(t) & =\left(\frac{1}{p(t)}-g(t)\right) r \sin \theta \cos \theta \\
\theta^{\prime}(t) & =\frac{1}{p(t)} \cos ^{2} \theta+g(t) \sin ^{2} \theta .
\end{aligned}
$$

In the sequel we use the above transformed system to prove the following theorems related to the solutions of (2.1). The first two results are from $[6]$.

Theorem 2.5 (Oscillation Theorem). Suppose $p_{i}^{\prime}, g_{i}$ are piecewise continuous functions in $[a, b]$, and $\mathcal{L}_{i} x=\left(p_{i} x^{\prime}\right)^{\prime}+g_{i} x=0, i=1,2$. Let $0<p_{2}(t) \leq p_{1}(t), g_{2}(t) \geq g_{1}(t)$. If $\mathcal{L}_{1} \phi_{1}=0, \mathcal{L}_{2} \phi_{2}=0$, where $\phi_{i}$ are solutions of $\mathcal{L}_{i} x$, and $\omega_{2}(a) \geq \omega_{1}(a)$, where $\omega_{i}$ are solutions of (2.4), then $\omega_{2}(t) \geq \omega_{1}(t) \forall t \in(a, b)$ (1). Moreover, if $g_{2}(t)>g_{1}(t), t \in(a, b)$, then $\omega_{2}(t)>\omega_{1}(t), \forall t \in(a, b]$

Proof. From (2.4), $\omega_{i}^{\prime}=\frac{1}{p_{i}} \cos ^{2} \omega_{i}+g_{i} \sin ^{2} \omega_{i}, i=1,2$. We have

$$
\left(\omega_{2}-\omega_{1}\right)=\left(g_{1}-\frac{1}{p_{1}}\right)\left(\sin ^{2} \omega_{2}-\sin ^{2} \omega_{1}\right)+h
$$

where

$$
h=\left(\frac{1}{p_{1}}-\frac{1}{p_{2}}\right) \cos ^{2} \omega_{2}+\left(g_{2}-g_{1}\right) \sin ^{2} \omega_{2} .
$$

Note that $h \geq 0$. 
If $\omega_{2}-\omega_{1}=u$, then by $(3), u^{\prime}=f u+h$, where

$$
f=\left(g_{1}-\frac{1}{p_{1}}\right)\left(\sin \omega_{1}+\sin \omega_{2}\right) \frac{\sin \omega_{2}-\sin \omega_{1}}{\omega_{2}-\omega_{1}} .
$$

Hence, $\mathrm{f}$ is a piecewise continuous and uniformly bounded function. Since $h \geq 0, u^{\prime}-f u \geq 0$. Set $F(t)=\int_{t}^{a} f(s) d s$. Then $e^{F} u^{\prime}+$ $F^{\prime} e^{F} u \geq 0$, and by integrating this,

$$
e^{F(t)} u(t) \geq e^{F(a)} u(a) \geq 0 \quad(4) .
$$

Now it is easy to see that the above proves (1).

Now suppose that (2) fails to hold. We show that there exist some $c>a$ such that $\omega_{2}(t)=\omega_{1}(t)(a \geq t \geq c) \quad$ (5).

Suppose not, then by (1) there exists a sequence $\left\{t_{j}\right\}_{j=1}^{n}$ such that $a$ is a limit point of it with $\omega_{2}\left(t_{j}\right)>\omega_{1}\left(t_{j}\right), j=1 \ldots n$. Now using (4) with $a$ replaced by $t_{j}$, it follows that for $t>t_{j}, \omega_{2}(t)>\omega_{1}(t)$, $j=1 \ldots n$. With $t_{j}$ arbitrarily close to $a$ we have that (2) implies (5). This leads to a contradiction.

Using (5), (3) is true with $g_{2}>g_{1}$ only if $\omega_{2}=\omega_{1}=0(\bmod \pi)$, and if $p_{1}=p_{2}$ in $(\mathrm{a}, \mathrm{c})$. However, since $\omega_{i}, i=1 \ldots n$, are solutions of (2.4), the case $\omega_{1}=\omega_{2}=0(\bmod \pi)$ in $(\mathrm{a}, \mathrm{c})$ is impossible. This proves (2) if $g_{1}>g_{2}$, and concludes the proof of the theorem.

Theorem 2.6 (Sturm Comparison). Suppose $\phi$ is a real solution of $\left(p x^{\prime}\right)^{\prime}+g_{1} x=0$ and $\psi$ is a real solution of $\left(p x^{\prime}\right)^{\prime}+g_{2} x=0$, where $x \in(a, b)$. Let $g_{1}(t)>g_{2}(t)$ in $(a, b)$. If $t_{1}, t_{2}$ are successive zeros of $\phi$ in $(a, b)$, then $\psi$ must vanish in some point in $\left(t_{1}, t_{2}\right)$.

Proof.

Claim 2.7. $\phi(t)$ can vanish only where $\omega(t)=k \pi, k \in \mathbb{Z}$ (where $\omega(t)$ is a solution of (2.4).)

Proof. For a solution $\phi$ of (2.1) there is a solution $r=\varrho(t), \theta=\omega(t)$ of $(2.3),(2.4)$ respectively, where $\varrho^{2}=\left(p \phi^{\prime}\right)^{2}+\phi^{2}, \omega=\arctan \left(\frac{\phi}{p \phi^{\prime}}\right)$. Since $\phi$ and $\phi^{\prime}$ do not vanish simultaneously, it follows that $\varrho^{2}(t)>0$, and without loss of generality we can assume that $\varrho(t)>0$. A consequence of this is that $\phi(t)=\varrho(t) \sin \omega(t)$ can vanish only where $\omega(t)=k \pi, k \in \mathbb{Z}$.

Now since $\cos ^{2} \theta, \sin ^{2} \theta$ are uniformly bounded, (2.4) has a solution over any interval on which $p>0$ and $\mathrm{p}, \mathrm{g}$ are piecewise continuous functions (Picard Theorem). Since the right hand side of (2.4) is 
differentiable in $\theta$, it follows that the solution is unique in the usual sense. Now the proof of the theorem follows directly from the claim, the monotonicity of $\omega(t)$, and Theorem 2.5.

The following theorem is from [3].

Theorem 2.8 (Disconjugacy). Consider the problem

$$
\mathcal{L} x=\left(p(t) x^{\prime}(t)\right)^{\prime}+g(t) x(t)=0,
$$

where $t \in[a, \infty), x(a)=0$, and without loss of generality $x^{\prime}(a)>0$. If $p(t), g(t)$ are continuous in $[a, \infty)$, and $\int_{a}^{\infty}\left(\frac{1}{p(t)}+|g(t)|\right) d t \leq \pi$ with $p(t)>0, t \in[a, \infty)$, then no nontrivial solution of $\mathcal{L} x=0$ has two zeros in $[a, \infty)$.

Proof. Recall that $\theta^{\prime}(t)=\frac{1}{p(t)} \cos ^{2} \theta+g(t) \sin ^{2} \theta, \theta(a)=0$, and integrate it to obtain

$\theta(s)=\int_{a}^{s}\left(\frac{1}{p(t)} \cos ^{2} \theta(t)+g(t) \sin ^{2} \theta(t)\right) d t \leq \int_{a}^{s}\left(\frac{1}{p(t)}+|g(t)|\right) d t$.

Hence,

$$
\theta(s)<\int_{a}^{\infty}\left(\frac{1}{p(t)}+|g(t)|\right) d t \leq \pi .
$$

Since $\theta^{\prime}(a)>0, \theta(t)<\int_{a}^{\infty}\left(\frac{1}{p(t)}+|g(t)|\right) d t \leq \pi, t \in[a, \infty)$. Since the zeros of $\mathcal{L} x=0$ occur when $\theta(t)=k \pi, k \in \mathbb{Z}$, the above inequality proves the theorem.

Theorem 2.10. Consider the problem $\mathcal{L} x=\left(p(t) x^{\prime}(t)\right)^{\prime}+g(t) x(t)=$ $0, x(a)=0\left(x^{\prime}(a)>0\right)$, where $g(t)<0, \forall t \in[a, \infty)$. Then the nontrivial solution of $\mathcal{L} x=0$ has at most one zero in $[a, \infty)$.

Proof. We know that $\theta(a)=0$ and $\theta^{\prime}(a)>0$. Now the monotonicity of $\theta(t)$ implies that for some $b \in(a, \infty), \theta(b)=\frac{\pi}{2}$. Since $\theta^{\prime}(b)=\frac{1}{p(t)} \cos ^{2} \theta(b)+g(t) \sin ^{2} \theta(b)=g(b)$, we get that $0<\theta(t)<$ $\frac{\pi}{2}$ in $(a, \infty)$. Therefore in $(a, \infty)$ there are no zeros of any nontrivial solution of $\mathcal{L} x=0$; since if they were any, they would occur at $\theta(t)=k \pi$. This concludes the proof.

In the following theorem, where its proof is taken from [9], we will make use of a modified Prüfer transformation in order to give an important result about the distance between two successive zeros of a fixed nontrivial solution of the equation

$$
x^{\prime \prime}(t)+p_{1}(t) x^{\prime}(t)+p_{2}(t) x(t)=0,
$$


where $p_{1}(t), p_{2}(t)$ are piecewise continuous, real valued functions in a closed interval.

Theorem 2.12. Let $a$ and $b$ be consecutive zeros of a fixed nontrivial solution of (2.11), and let $\gamma$ be a differentiable function defined on $[a, b]$. Set

$$
\begin{gathered}
M_{1} \equiv \sup _{a \leq t \leq b}\left(\left|2 \gamma(t)-p_{1}(t)\right|\right) \\
M_{2} \equiv \sup _{a \leq t \leq b}\left(\left|\gamma^{\prime}(t)-p_{2}(t)-\gamma^{2}(t)+p_{1}(t) \gamma(t)\right|\right) .
\end{gathered}
$$

Then

$$
b-a \geq 2 \int_{0}^{\infty} \frac{d s}{1+M_{1} s+M_{2} s^{2}} .
$$

Proof. Let $x$ denote the solution referred to the statement of the theorem. Without loss of generality assume that $x(t)>0 \forall t \in(a, b)$, and that $x^{\prime}(a)>0, x^{\prime}(b)<0$. Define the real valued functions $R$ and $\Theta$ by the relations:

$$
\begin{gathered}
R \sin \Theta=x \\
R \cos \Theta=x^{\prime}+\gamma x
\end{gathered}
$$

where $R(t)>0, \Theta(t) \in[0, \pi], t \in[a, b]$. We differentiate (1), (2) and substitute into (2.11). Then

$$
\begin{gathered}
R^{\prime} \cos \Theta-R \Theta^{\prime} \sin \Theta=R\left(\gamma-p_{1}\right) \cos \Theta+R\left(\gamma^{\prime}-p_{2}-\gamma^{2}+p_{1} \gamma\right) \sin \Theta \\
R^{\prime} \sin \Theta+R \Theta^{\prime} \cos \Theta=R \cos \Theta-R \gamma \sin \Theta \quad(4) .
\end{gathered}
$$

We eliminate $R^{\prime}$ from (3),(4), and we have

$\Theta^{\prime}=\cos ^{2} \Theta-\left(2 \gamma-p_{1}\right) \sin \Theta \cos \Theta+\left(\gamma^{\prime}-p_{2}-\gamma^{2}+p_{1} \gamma\right) \sin ^{2} \Theta$

From (1) we observe that the zeros of $x$ occur when $\Theta(t)=k \pi$, and from (5) we note that $\Theta$ is increasing at $k \pi$ since $\Theta^{\prime}(k \pi)=1$. Hence we can suppose that $\Theta(a)=0$ and $\Theta(b)=\pi$. We use the intermediate value theorem to obtain $t \in(a, b)$ such that $\Theta(t)=\frac{\pi}{2}$. Now let $\alpha$ denote the least such $t$. For $t \in(a, \alpha), \sin \Theta$ and $\cos \Theta$ are both positive. Now we use (5) to get

$$
\left|\Theta^{\prime}\right| \leq \cos ^{2} \Theta+M_{1} \sin \Theta \cos \Theta+M_{2} \sin ^{2} \Theta,
$$


and so

$$
\begin{array}{r}
\alpha-a \geq \int_{o}^{\frac{\pi}{2}} \frac{d \Theta}{\cos ^{2} \Theta+M_{1} \sin \Theta \cos \Theta+M_{2} \sin ^{2} \Theta}= \\
\int_{0}^{\infty} \frac{d s}{1+M_{1} s+M_{2} s^{2}}
\end{array}
$$

Similarly, if $\beta$ denotes the largest $t \in(a, b)$ such that $\Theta(t)=k \pi$, then

$$
b-\beta \geq \int_{0}^{\infty} \frac{d s}{1+M_{1} s+M_{2} s^{2}}
$$

By combining (6) and (7) we have

$$
b-a \geq 2 \int_{0}^{\infty} \frac{d s}{1+M_{1} s+M_{2} s^{2}} .
$$

By choosing appropriate values for $\gamma$ and imposing certain conditions on $p_{1}(t)$ and $p_{2}(t)$, we can derive some very remarkable results.

Corollary 2.13. If $p_{1} \equiv 0$, then $\sup _{a \leq t \leq b}\left|\int_{a}^{t} p_{2}(s) d s\right| \geq \frac{2}{b-a}$.

Proof. Set $\gamma \equiv \int_{a}^{t} p_{2}(s) d s$. Thus,

$$
\begin{aligned}
& M_{1}=2 \sup _{a \leq t \leq b}\left|\int_{a}^{t} p_{2}(s) d s\right|, \\
& M_{2}=\sup _{a \leq t \leq b}\left|\int_{a}^{t} p_{2}(s) d s\right|^{2} .
\end{aligned}
$$

Let $M=\sup _{a \leq t \leq b}\left|\int_{a}^{t} p_{2}(s) d s\right|$. By Theorem 2.12 we have

$$
b-a \geq 2 \int_{0}^{\infty} \frac{d t}{1+2 M t+M^{2} t^{2}}=\frac{2}{M} .
$$

Corollary 2.14. If $p_{1}(t)$ is differentiable then

$$
\sup _{a \leq t \leq b}\left|\frac{p_{1}^{\prime}}{2}-p_{2}+\frac{p_{1}^{2}}{4}\right| \geq \frac{\pi^{2}}{(b-a)^{2}} .
$$

Proof. Choose $\gamma \equiv \frac{p_{1}}{2}$. Then $M_{1}=0$ and

$$
M_{2}=\sup _{a \leq t \leq b}\left|\frac{p_{1}^{\prime}}{2}-p_{2}+\frac{p_{1}^{2}}{4}\right| \text {. }
$$


From Theorem 2.12,

$$
b-a \geq 2 \int_{o}^{\infty} \frac{d t}{1+M_{2} t^{2}}=\pi \frac{1}{\sqrt{M_{2}}} .
$$

Similarly we can prove that if $p_{1}$ is differentiable, then

$$
\sup _{a \leq t \leq b}\left|\frac{p_{1}(a)}{2}-p_{1}(t)+\int_{a}^{t}\left(p_{2}(s)-\frac{p_{1}(s)^{2}}{4}\right) d s\right| \geq \frac{2}{b-a} .
$$

(To see the above, choose $\gamma \equiv \frac{p_{1}(t)}{2}+\int_{a}^{t}\left(p_{2}-\frac{p_{1}^{\prime}}{2}-\frac{p_{1}^{2}}{4}\right) d s$ and apply Theorem 2.12.)

\section{Bounds of Solutions And Asymptotic Behavior}

In this section we will use the Prüfer transformation in order to study the asymptotic behavior of solutions of the equation

$$
\mathcal{L} x=\left(p(t) x^{\prime}\right)^{\prime}+g(t) x=0 \text { when } t \rightarrow \infty,
$$

and we will prove that every solution of $\mathcal{L} x=0$ is bounded if

$$
\int_{a}^{\infty}\left|\frac{1}{p(t)}-g(t)\right| d t<\infty
$$

In the proof of the following theorem we will use Gronwall's Lemma.

Lemma 3.1 (Gronwall's Lemma). If $u, v$ are real valued nonnegative functions in $L_{1}(m)$ with domain $\left\{t: t \geq t_{0}\right\}$, and if there exists a constant $M \geq 0$ such that for every $t \geq t_{0}$

$$
u(t) \leq M+\int_{t_{0}}^{t} u(s) v(s) d s,
$$

then

$$
u(t) \leq M \exp \left(\int_{t_{0}}^{t} v(s) d s\right)
$$

The following theorem is from [3].

Theorem 3.2. Every solution $x(t)$ of $\mathcal{L} x=0$ satisfies the inequality

$$
|x(t)| \leq K \exp \left[\frac{1}{2} \int_{a}^{t}\left|\frac{1}{p(s)}-g(s)\right| d s\right],
$$

where $t \in(a, \infty)$ and $K=\sqrt{x^{2}(a)+\left(p(a) x^{\prime}(a)\right)^{2}}$. Moreover, if

$$
\int_{a}^{\infty}\left|\frac{1}{p(t)}-g(t)\right| d t<\infty
$$


then every solution of $\mathcal{L} x=0$ is bounded.

Proof. We will use once more the transformed system

$$
\begin{gathered}
r^{\prime}(t)=\left[\frac{1}{p(t)}-g(t)\right] r(t) \sin \Theta(t) \cos \Theta(t) \\
\Theta^{\prime}(t)=\frac{1}{p(t)} \cos ^{2} \Theta(t)+g(t) \sin ^{2} \Theta(t)
\end{gathered}
$$

where $x(t)=r(t) \sin \Theta(t)$, to conclude that

$$
|x(t)| \leq|r(t)| \quad(3)
$$

From (1) we have that

$$
r(s)-r(a)=\int_{a}^{s}\left[\frac{1}{p(t)}-g(t)\right] r(t) \frac{1}{2} \sin 2 \Theta(t) d t, \quad s \in(a, \infty) .
$$

Hence,

$$
|r(t)| \leq r(a)+\frac{1}{2} \int_{a}^{s}\left|\frac{1}{p(t)}-g(t)\right||r(t)| d t .
$$

We apply Gronwall's Lemma in the above inequality and we get

$$
|r(s)| \leq r(a) \exp \left(\frac{1}{2} \int_{a}^{s}\left|\frac{1}{p(t)}-g(t)\right| d t\right),
$$

and so from (3),

$$
|x(t)| \leq r(a) \exp \left(\frac{1}{2} \int_{a}^{t}\left|\frac{1}{p(s)}-g(s)\right| d s\right) .
$$

Now observe that if $\int_{a}^{\infty}\left|\frac{1}{p(t)}-g(t)\right| d t<\infty$, then

$$
\exp \left[\frac{1}{2} \int_{a}^{\infty}\left|\frac{1}{p(t)}-g(t)\right| d t\right] \in \mathbb{R} .
$$

Thus if $M=r(a) \exp \left(\frac{1}{2} \int_{a}^{\infty}\left|\frac{1}{p(t)}-g(t)\right| d t\right)$, then $M \in \mathbb{R}$ and $|x(t)| \leq$ $M$. This shows that $x(t)$ is bounded.

Now we will study the asymptotic behavior of the solutions of the equation

$$
x^{\prime \prime}(t)+(1+g(t)) x(t)=0,
$$

where if $x_{0}$ is a fixed real number that is sufficiently "small" for large values of $x$, then $g(t)$ is a real continuous function for $x \geq x_{0}$. Now observe that (3.3) is of the standard form $\mathcal{L} x=\left(x^{\prime} p\right)^{\prime}+g x=0$, where $p(t) \equiv 1, g(t) \mapsto g(t)+1$. In (3.3) we will use a modified Prüfer 
transformation by substituting $\Theta(t)$ with $\Theta(t)+1$. The transformed equations are

$$
\begin{gathered}
r^{\prime}(t)=-g(t) r(t) \sin (t+\Theta(t)) \cos (t+\Theta(t)) \\
(t+\Theta(t))^{\prime}=1+g(t) \sin ^{2}(t+\Theta(t)),
\end{gathered}
$$

which yield to

$$
\begin{gathered}
\frac{r^{\prime}(t)}{r(t)}=-\frac{1}{2} g(t) \sin 2(t+\Theta(t)), \\
\Theta^{\prime}(t)=\frac{1}{2} g(t)(1-\cos 2(t+\Theta(t)) .
\end{gathered}
$$

Using the above transformed system we will prove the following theorem which asserts that the fundamental system of solutions $x_{1}, x_{2}$ of (3.3) when $t \rightarrow \infty$ is

$$
\left\{\begin{array}{l}
x_{1}(t)=\cos (t)+o(1) \quad x_{2}(t)=\sin (t)+o(1) \\
x_{1}^{\prime}(t)=-\sin (t)+o(1) \quad x_{2}^{\prime}=\cos (t)+o(1) .
\end{array}\right.
$$

The following theorem is from [8].

Theorem 3.7 (Asymptotic Behavior). Let $g(t)$ be a real continuous function for $t \geq t_{0}$, where $t_{0}$ is a fixed real number, and assume that the following integrals exist:

$$
\left\{\begin{array}{l}
\int_{t}^{\infty} g(s) d s, g_{1}(s)=\int_{t}^{\infty} g(s) \cos (2 s) d s \\
g_{2}(t)=\int_{t}^{\infty} g(s) \sin (2 s) d s, \int_{t_{0}}^{\infty}\left|g(t) g_{j}(t)\right| d t, \quad j=1,2 .
\end{array}\right.
$$

Then the equation $x^{\prime \prime}+(1+g(t) x)=0$ has a fundamental system of solutions satisfying (3.6).

Note that the above assumptions are certainly satisfied if $g \in$ $L\left[t_{0}, \infty\right]$.

Proof. Step 1: We will show that for any nontrivial solution of (3.3) the corresponding $\Theta(t), r(t)$, given by (3.4) and (3.5) respectively, tend to finite limits as $t \rightarrow \infty$. By using

$$
g(t) \cos (2 t) \cos (2 \Theta)=-\left(g_{1} \cos 2 \Theta\right)^{\prime}-2 g_{1} \Theta^{\prime} \sin 2 \Theta
$$

and

$$
g(t) \sin (2 t) \sin (2 \Theta)=-\left(g_{2} \sin 2 \Theta\right)^{\prime}-2 g_{2} \Theta^{\prime} \cos 2 \Theta,
$$

(3.5) can be written as:

$\Theta^{\prime}(t)=\frac{1}{2} g+\frac{1}{2}\left(g_{1} \cos 2 \Theta\right)^{\prime}-\frac{1}{2}\left(g_{2} \sin 2 \Theta\right)^{\prime}+g_{1} \Theta^{\prime} \sin 2 \Theta+g_{2} \Theta^{\prime} \cos 2 \Theta$. 
Since by (3.5) $\left|\Theta^{\prime}\right| \leq|g|$, it follows from the hypothesis of the theorem that $\Theta^{\prime}$ is integrable over $\left[x_{0}, \infty\right)$. Now from (3.9) we have

$$
\left|\Theta^{\prime}(t)\right|=\frac{1}{2}|g|+\frac{1}{2}\left|\left(g_{1} \cos 2 \Theta\right)^{\prime}\right|+\frac{1}{2}\left|\left(g_{2} \sin 2 \Theta\right)^{\prime}\right|+\left|g_{1} g\right|+\left|g_{2} g\right| .
$$

From (3.8) and (3.9) we conclude that $\Theta(t)$ tends to a finite limit as $t \rightarrow \infty$. Similarly using the relations

$$
\begin{aligned}
& g(t) \sin 2 t \cos 2 t=-\left(g_{2} \cos 2 \Theta\right)^{\prime}-2 g_{2} \Theta^{\prime} \sin 2 \theta \\
& g(t) \cos 2 t \sin 2 t=-\left(g_{1} \sin 2 \Theta\right)^{\prime}-2 g_{1} \Theta^{\prime} \cos 2 \theta,
\end{aligned}
$$

we can write (3.4) as

$\frac{r^{\prime}}{r}=(1 / 2)\left(g_{2} \cos 2 \Theta\right)^{\prime}+(1 / 2)\left(g_{1} \sin 2 \Theta\right)^{\prime}+g_{2} \Theta^{\prime} \sin 2 \theta-g_{1} \Theta^{\prime} \cos 2 \Theta$.

Since $(\log r)^{\prime}=\frac{r^{\prime}}{r}$, from (3.4) we get $\left|(\log r)^{\prime}\right| \leq|g|$, and so $(\log r)^{\prime}$ is integrable over $\left[x_{0}, \infty\right]$. Now using (3.10) we obtain

$$
\left|(\log r)^{\prime}\right| \leq(1 / 2)\left|\left(g_{2} \cos 2 \Theta\right)^{\prime}\right|+(1 / 2)\left|\left(g_{1} \sin 2 \Theta\right)^{\prime}\right|+\left|g_{1} g\right|+\left|g_{2} g\right|,
$$

and hence by (3.8), $\log r$ tends to a finite limit as $t \rightarrow \infty$. Therefore $r$ has a positive (finite) limit. This concludes the proof of Step 1.

Step 2: Now we will show that two distinct solutions of (3.3) cannot tend to the same limit as $t \rightarrow \infty$. If we integrate (3.9), then by (3.5) we get

$$
\begin{aligned}
\Theta(t)= & \Theta(\infty)+1 / 2 \int_{t}^{\infty} g(s) d s+1 / 2\left(g_{1} \cos 2 \Theta-g_{2} \sin 2 \Theta\right) \\
& -1 / 2 \int_{t}^{\infty}\left[g\left(g_{1} \sin 2 \Theta\right)+g_{2} \cos 2 \Theta\right)(1-\cos 2(s+\Theta)] d s .
\end{aligned}
$$

Now choose $t_{1}$ large enough such that $\left|g_{1}(t)\right| \leq 1 / 16$ for every $t \geq t_{1}$ and $\int_{t_{1}}^{\infty}\left|g_{j} g\right| d s \leq 1 / 16$, where $j=1,2$. If $\hat{\Theta}(t)$ is a solution of (3.5) with the same limit as $\Theta$ and $\Theta \neq \hat{\Theta}$, then if we subtract from (3.11) the corresponding relation with $\Theta$ replaced by $\hat{\Theta}$, we get that for $t \geq t_{1}$

$$
|\Theta(t)-\hat{\Theta}(t)| \leq 1 / 2 \sup _{s \geq t_{1}}|\Theta(s)-\hat{\Theta}(s)| .
$$

Hence $\sup _{s>t_{1}}|\Theta(s)-\hat{\Theta}(s)|=0$, and thus, $\Theta=\hat{\Theta}$. This is clearly a contradiction. Similarly we have $r(t)=\hat{r}(t)$. This conclude the proof of step 2 . 
In terms of (3.3), this means that for any nontrivial solution $x$ there exist constants $A, \alpha,(A>0,0 \leq \alpha<2 \pi)$ such that for $t \rightarrow \infty$

$$
\begin{aligned}
& \lim _{t \rightarrow \infty} x(t)=A \sin (t+\alpha)+o(1), \\
& \lim _{t \rightarrow \infty} x^{\prime}(t)=A \cos (t+\alpha)+o(1) .
\end{aligned}
$$

Moreover, if $x_{1}, x_{2}$ are linearly independent solutions of (3.3), then the corresponding phase shifts $\alpha_{1}, \alpha_{2}$ cannot differ by an integer multiple of $\pi$. Consequently, by forming suitable combinations of $x_{1}, x_{2}$, we can obtain solutions with asymptotic behavior as it is described in (3.6).

Examples 3.12. (1). Given the equation $y^{\prime \prime} \pm k y=0, k>0$, from Theorem 2.6 with $p(t)=1, g(t)= \pm k$, we get

$$
|y(t)| \leq \sqrt{y^{2}(a)+\left(y^{\prime}\right)^{2}(a)} \exp (1 / 2|1 \pm k|) .
$$

(2). Consider the equation $\left(p(t) y^{\prime}\right)^{\prime}+\frac{k}{p(t)} y=0$, where $\frac{1}{p(t)} \in L^{1}(m)$ and $k>0$. Then by Theorem 3.2 , since

$$
\int_{a}^{\infty}\left|\frac{1-k}{p(t)}\right| d t \leq|1-k| \int_{a}^{\infty} \frac{d t}{|p(t)|}<\infty
$$

we conclude that the solutions $y(t)$ are bounded.

(3). In this example we will illustrate an application of Theorem 3.7. We will study the asymptotic behavior of the equation

$$
x^{\prime \prime}(t)+\left(1+\frac{\sin 2 t}{t}\right) x(t)=0, t \geq 0, \lambda \in(\mathbb{Z} \backslash\{ \pm 2\}) .
$$

Consider the function $g(t)=\frac{\sin \lambda t}{t}, \lambda \neq \pm 2, \lambda \in \mathbb{Z}$. Observe that $g(t) \notin L^{1}(m)$, but the hypothesis of the Theorem 3.7 are satisfied. Indeed,

$$
\begin{aligned}
\int_{a}^{\infty}\left|\frac{\sin \lambda s}{s}\right| d s & \geq \int_{a}^{\infty} \frac{\sin ^{2} \lambda s}{|s|} d s \\
& =\int_{a}^{\infty} \frac{(1-\cos 2 \lambda s)}{|2 s|} d s \\
& =1 / 2 \int_{2 a}^{\infty} \frac{1-\cos u}{|u|} d u \\
& =1 / 2 \int_{2 a}^{\infty} \frac{d u}{u}-1 / 2 \int_{2 a}^{\infty} \frac{\cos u}{u} d u
\end{aligned}
$$


Moreover, $\int_{2 a}^{\infty} \frac{d u}{u}=\infty$ and $\int_{2 a}^{\infty} \frac{\cos u}{u} d u<\infty$, hence $\int_{a}^{\infty}\left|\frac{\sin \lambda s}{s}\right| d s=$ $\infty$. This shows that $g(t) \notin L^{1}(m)$.

Observe that $\int_{t}^{\infty} g(s) d s=\int_{t}^{\infty} \frac{\sin \lambda s}{s} d s$ exists, since if $0<s<t$,

$$
\left|\int_{s}^{t} \frac{\sin \lambda s}{s} d s\right| \leq\left|\frac{\cos \lambda s}{\lambda s}-\frac{\cos \lambda t}{\lambda t}-\frac{1}{\lambda} \int_{s}^{t} \frac{\cos \lambda s}{s} d s\right| .
$$

Thus,

$$
\left|\int_{s}^{t} \frac{\sin \lambda s}{s} d s\right| \leq 1 /|\lambda|\left(1 / s+1 / t+\int_{s}^{t} \frac{1}{s^{2}} d s\right)=\frac{2}{|\lambda| s} .
$$

Additionally,

$$
g_{1}(t)=\int_{t}^{\infty} \frac{\sin \lambda s}{s} \cos 2 s d s<\infty
$$

(see [15], p.96 (15.34)) and

$$
g_{2}(t)=\int_{t}^{\infty} \frac{\sin \lambda s}{s} \sin 2 s d s<\infty
$$

(see [15], p.96 (15.38)). Moreover, since $g^{2} \in L^{1}(m)$, it is elementary to show that $\int_{a}^{\infty}\left|g g_{j}\right| d t<\infty$ for $a>0, j=1,2$. This shows that the hypothesis of the theorem are satisfied, and thus the equation

$$
x^{\prime \prime}(t)+\left(1+\frac{\sin 2 t}{t}\right) x(t)=0, t \geq 0, \lambda \in(\mathbb{Z} \backslash\{ \pm 2\})
$$

has a fundamental system of solutions satisfying (3.6).

Remark 3.14. If $\lambda= \pm 2$ we can easily see that

$$
\int_{a}^{\infty} \frac{\sin ^{2} \lambda s}{s} d s=\int_{a}^{\infty} \frac{1-\cos 4 s}{2} d s=\int_{a}^{\infty} \frac{d s}{2}-\int_{a}^{\infty} \frac{\cos 4 s}{2} d s
$$

where $\int_{a}^{\infty} \frac{d s}{2}=\infty$ and $\int_{a}^{\infty} \frac{\cos 4 s}{2} d s<\infty \forall a \in[0, \infty)$. This shows that

$\int_{a}^{\infty} \frac{\sin ^{2} \lambda s}{s} d s=\infty$ and thus the integral $g_{2}(t)=\int_{t}^{\infty} \frac{\sin \lambda s}{s} \sin 2 s d s$ does not exist and the Theorem 3.7 does not apply. 


\section{Spectral Theory}

In this section we will consider the regular Sturm-Liouville eigenvalue problem with boundary conditions. We use once more "Prüfer transformation" techniques to obtain theorems concerning the spectrum of such problems.

Consider the system

$$
\left\{\begin{array}{l}
\left(p(t) x^{\prime}(t)\right)^{\prime}+(\lambda r(t)-q(t)) x(t)=0, t \in[a, b], \\
A x(a)-B x^{\prime}(a)=0 \\
\Gamma x(b)-\Delta x^{\prime}(b)=0 .
\end{array}\right.
$$

There is no loss of generality in assuming that $0 \leq|A| \leq 1,0 \leq$ $B / p(a) \leq 1$ and $\frac{A^{2}+B^{2}}{p^{2}(a)}=1$. This means that there is a unique constant $\alpha, 0 \leq \alpha \leq \pi$, such that the expression $A x(a)-B x^{\prime}(a)=0$ can be written as $(\cos \alpha) x(a)-(\sin \alpha) p(a) x^{\prime}(a)=0$. Similarly, there is a unique constant $\beta, 0 \leq \beta \leq \pi$, such that $\Gamma x(b)-\Delta x^{\prime}(b)=0$ can be written as $(\cos \beta) x(\beta)-(\sin \beta) p(b) x^{\prime}(b)=0$. Hence the above system is equivalent to the following system

$$
\left\{\begin{array}{l}
\left(p(t) x^{\prime}(t)\right)^{\prime}+(\lambda r(t)-q(t)) x(t)=0, t \in[a, b], \lambda \neq 0, \\
(\cos \alpha) x(a)-(\sin \alpha) p(a) x^{\prime}(a)=0 \\
(\cos \beta) x(\beta)-(\sin \beta) p(b) x^{\prime}(b)=0
\end{array}\right.
$$

where $\lambda$ is a real parameter and $p^{\prime}, r, q$ are real and piecewise continuous functions in $[a, b]$ with $p>0, r>0$ in $[a, b]$. The values of $\lambda$ for which the system (4.1) has a nontrivial solution are called eigenvalues and the corresponding (nontrivial) solutions, eigenfunctions.

Next, we present the most important theorem (which is taken from [4]) about the eigenvalues and the zeros of eigenfunctions of (4.1).

Theorem 4.2. There is an infinite number of eigenvalues $\lambda_{0}, \lambda_{1}$, $\lambda_{2}, \ldots$ forming a monotone increasing sequence with $\lambda_{n} \rightarrow \infty$ as $n \rightarrow \infty$ of (4.1). Moreover, the eigenfunctions $\phi_{n}$ corresponding to $\lambda_{n}$ have exactly $n$ zeros in $(a, b)$. Note that by Theorem 2.6 (Sturm Comparison) the zeros of $\phi_{n}$ separate those of $\phi_{n+1}$.

Proof. Let $\phi(t, \lambda)$ be the unique solution of the first equation of (4.1) which satisfies $\phi(a, \lambda)=\sin \alpha, \phi^{\prime}(a, \lambda)=\cos \alpha$. Then $\phi$ satisfies the second equation of (4.1). Let $r(t, \lambda), \omega(t, \lambda)$ be the corresponding Prüfer transformations of $\phi(t, \lambda)$. The initial conditions are transformed to $r(a, \lambda)=1, \omega(a, \lambda)=\alpha$. Eigenvalues are those values of 
$\lambda$ for which $\phi(t, \lambda)$ satisfies the third equation of (4.1). That is, are those values of $\lambda$ for which $\omega(b, \lambda)=\beta+n \pi, n \in \mathbb{Z}$. By Theorem 2.5 (Oscillation) for any fixed $t \in[a, b], \omega(t, \lambda)$ is monotone and increasing in $\lambda$. Note that $\omega(t, \lambda)=0(\bmod \pi)$ if and only if $\phi(t, \lambda)=0$. From $\theta^{\prime}=\frac{1}{p} \cos ^{2} \theta+(\lambda r-q) \sin ^{2} \theta$ it is clear that $\theta^{\prime}=\frac{1}{p}>0$ at a zero of $\phi$, and hence $\omega(t, \lambda)$ is strictly increasing in a neighborhood of a zero.

Claim 4.3. For any fixed $t=c, c \in[a, b], \lim _{\lambda \rightarrow \infty} \omega(c, \lambda)=\infty$.

Proof. Since $\alpha \geq 0$ and since $\omega^{\prime}>0$ for $\omega=0(\bmod \pi), \omega(t, \lambda) \geq 0$. Thus it suffices to show that for some $t_{0}, \alpha<t_{0}<c$,

$$
\lim _{\lambda \rightarrow \infty}\left[\omega(c, \lambda)-\omega\left(t_{0}, \lambda\right)\right]=\infty .
$$

Let $t_{0}=\frac{a+b}{2}$, and $P, Q, R$ be constants such that over $\left(t_{0}, c\right), p(t) \leq$ $P, r(t) \geq R>0$ and $q(t) \leq Q$. Then the equation

$$
P x^{\prime \prime}+(\lambda R-Q)=0
$$

with solution $\hat{\phi}$ satisfying $\hat{\phi}\left(t_{0}, \lambda\right)=\phi\left(t_{0}, \lambda\right), \hat{\phi}^{\prime}\left(t_{0}, \lambda\right)=\phi^{\prime}\left(t_{0}, \lambda\right)$, has $\hat{\omega}\left(t_{0}, \lambda\right)=\omega\left(t_{0}, \lambda\right)$, and hence by Theorem 2.5

$$
\omega(c, \lambda)-\omega\left(t_{0}, \lambda\right) \geq \hat{\omega}(c, \lambda)-\hat{\omega}\left(t_{0}, \lambda\right) .
$$

(4.4) implies that the successive zeros of $\hat{\phi}$ have spacing $\pi \sqrt{\frac{P}{\lambda R-Q}}$, and hence $\lim _{\lambda \rightarrow \infty} \pi \sqrt{\frac{P}{\lambda R-Q}}=0$. Then for any integer $j>1, \hat{\phi}$ will have $j$ zeros between $t_{0}$ and $c$ for $\lambda$ large enough. Thus, $\hat{\omega}(c, \lambda)-$ $\hat{\omega}\left(t_{0}, \lambda\right) \geq j \pi$. Since $j$ is arbitrary, by $(4.5), \lim _{\lambda \rightarrow \infty}[\omega(c, \lambda)-$ $\left.\omega\left(t_{0}, \lambda\right)\right]=\infty$. This proves the claim.

Claim 4.6. For fixed $t=c, c \in(a, b]$, we have $\lim _{\lambda \rightarrow-\infty} \omega(c, \lambda)=0$.

Proof. We will use the equation $\theta^{\prime}=\frac{1}{p} \cos ^{2} \theta+(\lambda r-q) \sin ^{2} \theta$. Choose $\delta>0$ sufficiently small such that $\alpha<\pi-\delta$. If $\delta \leq \omega \leq \pi-\delta, \lambda<0$, and if $0<P \leq p, 0<R \leq r, Q \geq|q|$, then $\omega^{\prime}<1 / p-|\lambda| R \sin ^{2} \delta+$ $Q \leq-\frac{\alpha-\delta}{c-\alpha}<0$ whenever $\lambda<\left[\frac{\alpha-\delta}{\alpha-c}-Q-1 / p\right] R \sin ^{2} \delta<0$. Hence $\omega(c, \lambda) \leq \delta$ for $-\lambda$ sufficiently large. Since $\delta$ is arbitrary, $\lim _{\lambda \rightarrow-\infty} \omega(c, \lambda)$ $=0$, and this proves the claim.

Now for $c=b, \lim _{\lambda \rightarrow-\infty} \omega(b, \lambda)=0$. Since $\beta>0$, and since $\omega(b, \lambda)$ is monotone and increasing in $\lambda$, it follows that there is a value $\lambda=\lambda_{o}$ for which $\omega\left(b, \lambda_{o}\right)=\beta$. Since $0 \leq \alpha<\pi$ and $\beta \leq \pi$, 
$0<\omega\left(t, \lambda_{o}\right)<\pi$ in $(a, b)$. Now from this we immediately obtain that the solution $\phi\left(t, \lambda_{o}\right)$ satisfies the third equation of (4.1) and in addition it does not vanish. Now let $\lambda$ increase beyond $\lambda_{o}$. Then there is a unique $\lambda_{1}$ for which $\omega\left(b, \lambda_{1}\right)=\beta+\pi$. Clearly, $\phi\left(t, \lambda_{1}\right)$ satisfies the third equation of (4.1) and has exactly one zero in $(a, b)$. If we continue in this manner, the $n^{\text {th }}$ eigenvalue is determined by $\omega\left(b, \lambda_{n}\right)=\beta+n \pi$ and the $n^{t h}$ corresponding eigenfunction has exactly $n$ zeros in $(a, b)$. This concludes the proof of the theorem.

A Prüfer transformation, in combination with one dimensional Sobolev inequality, can be used to derive upper and lower bounds for the spectrum (set of eigenvalues) of regular self adjoint second order eigenvalue problems.

For the next theorem consider the following eigenvalue problem.

Let $q$ be a real function in $L^{s}(a, b), s \geq 1$, and let $\lambda_{o}<\lambda_{1}<\lambda_{2}<$ $\ldots$ and $\phi_{o}, \phi_{1}, \phi_{2}, \ldots$ denote the eigenvalues and real orthonormal eigenfunctions (see Theorem 4.2) of

$$
-y^{\prime \prime}+q(x) y=\lambda y \quad y(a)=y(b)=0 .
$$

We introduce the notation $f_{+}(x) \equiv \max (f(x), 0)$ and $f_{-}(x) \equiv$ $f_{+}(x)-f(x)$ for a real function $f$.

The following theorem is taken from [5].

Theorem 4.8. Let $\lambda<\lambda_{n}$. Then the eigenvalues of (4.7) satisfy the following inequality,

$\lambda_{n} \leq \lambda+\left(\frac{\pi(n+1)}{2(b-a)}+\left[\frac{(n+1)^{2} \pi^{2}}{4(b-a)^{2}}+\frac{\int_{a}^{b}(\lambda-q(x))_{-} d x}{b-a}\right]^{1 / 2}\right)^{2}$

which implies that

$$
\lambda_{n} \leq \lambda+\frac{(n+1)^{2} \pi^{2}}{(b-a)^{2}}+2 \frac{\int_{a}^{b}(\lambda-q(x))_{-} d x}{b-a}
$$

Proof. In the equation $-\phi_{n}^{\prime \prime}+q \phi_{n}=\lambda_{n} \phi_{n}$ we apply the modified Prüfer transformation

$$
\phi_{n}=r \sin \theta, \quad \phi_{n}^{\prime}=\sqrt{\lambda_{n}-\lambda} r \cos \theta, \quad r(x)>0 .
$$

This yields to

$$
\theta^{\prime}=\sqrt{\lambda_{n}-\lambda} \cos ^{2} \theta-\frac{\left(q-\lambda_{n}\right) \sin ^{2} \theta}{\sqrt{\lambda_{n}-\lambda}}=\sqrt{\lambda_{n}-\lambda}-\frac{(q-\lambda) \sin ^{2} \theta}{\sqrt{\lambda_{n}-\lambda}}
$$


From (3) we have,

$$
\theta^{\prime} \geq \sqrt{\lambda_{n}-\lambda}-\frac{(\lambda-q)_{-} \sin ^{2} \theta}{\sqrt{\lambda_{n}-\lambda}} \geq \sqrt{\lambda_{n}-\lambda}-\frac{(\lambda-q)_{-}}{\sqrt{\lambda_{n}-\lambda}}
$$

Since $\phi_{n}$ has exactly $\mathrm{n}$ zeros in $(\mathrm{a}, \mathrm{b})$ and vanishes at $a, b$, (see Theorem 4.2) we may take $\theta(a)=0$ which implies that $\theta(b)=(n+1) \pi$. Now we integrate (4) to conclude that

$$
(n+1) \pi \geq(b-a) \sqrt{\lambda_{n}-\lambda}-\frac{\int_{a}^{b}(\lambda-q(x))-d x}{\sqrt{\lambda_{n}-\lambda}}
$$

This is equivalent to $A \leq B \sqrt{A}+C$, where

$A \equiv\left(\lambda_{n}-\lambda\right), B \equiv(b-a)^{-1}(n+1) \pi, C \equiv(b-a)^{-1} \int_{a}^{b}(\lambda-q(x))_{-} d x$.

Hence $\sqrt{A} \leq\left[B+\sqrt{B^{2}+4 C}\right] / 2$, which is equivalent to (1). Moreover $\sqrt{B^{2}+4 C}=B \sqrt{1+4 C / B^{2}} \leq B\left(1+2 C / B^{2}\right)=B+2 C / B$, since $\sqrt{1+x} \leq 1+x / 2 \forall x \geq 0$. Thus, $A \leq\left[B^{2}+2 B(B+2 C / B)+B^{2}+\right.$ $4 C] / 4 \leq B^{2}+2 C$, which is exactly (2). This concludes the proof.

Remark 4.9. The proof of Theorem 4.8 gives necessary and sufficient conditions for constructing $q$ which will make (1) equality. Equality holds in (1) if and only if equality holds in (5). Consequently,

$$
\int_{a}^{b}(\lambda-q(x))-\sin ^{2} \theta(x) d x=\int_{a}^{b}(\lambda-q(x))-d x,
$$

which implies that $(\lambda-q(x))_{-} \cos ^{2} \theta(x)=0$, a.e $[m]$. We integrate (3) and we get $(\lambda-q(x))+\sin ^{2} \theta(x)=0$, a.e $[m]$. Since $\theta^{\prime}(x)>0$, if $x=k \pi,(k \in \mathbb{Z})$, then $(\lambda-q(x))_{+}=0$, a.e $[m]$. Thus, $(\lambda-q(x))=$ $(\lambda-q(x))_{-}$and $(\lambda-q(x))_{-}=0$ a.e on $E$, where $E=\left\{x: \sin ^{2} \theta(x) \neq\right.$ $1\}$.

For example take $n=0$ and $\lambda=0$. Then $q(x) \geq 0$, and by (3) we have that

$$
q(x)= \begin{cases}\lambda_{o} & \{x: \theta(x)=\pi / 2\} \\ 0 & \text { elsewhere. }\end{cases}
$$

In the following theorems we are trying to find, under certain general conditions on the coefficient $q$, a best possible (optimal) upper bound on the real parameter $\lambda$ in order for the differential equation $y^{\prime \prime}(x)+$ $(\lambda-q(x)) y(x)=0, x \in[a, \infty)$, to have a nontrivial solution in $L^{2}(a, \infty)$. 
We consider the equation of the form

$$
y^{\prime \prime}+\left(f^{2}+f g+f k\right) y=0, x \in[a, \infty),
$$

where all quantities are real, subject to the following:

(i) $f(x)$ is positive, locally absolutely continuous in $[a, \infty)$, and satisfies

$$
\lim _{x \rightarrow \infty} f^{\prime}(x) f^{-2}(x)=0 .
$$

(ii) $g(x)$ is locally $L^{1}[a, \infty)$ and satisfies

$$
\begin{gathered}
\lim _{x \rightarrow \infty} g(x) f^{-1}(x)=0, \\
k(x) \in L^{1}(a, \infty) .
\end{gathered}
$$

We define

$$
\begin{gathered}
\psi_{1}(x)=\sup _{t \geq x}\left|f^{\prime}(t) / f^{2}(t)\right|, \\
\psi_{2}(x)=\sup _{t \geq x}|g(t) / f(t)|,
\end{gathered}
$$

and we assume that

$$
\psi_{1}^{2} f, \quad \psi_{2}^{2} f \text { are both in } L^{1}(a, \infty) .
$$

Then we have the following theorem (see [2]).

Theorem 4.17. Let the above (4.11) to (4.16) conditions hold, and let $y$ be a nontrivial solution for (4.10). Define $R(x)$ by

$$
R^{2}=f y^{2}+f^{-1}\left(y^{\prime}\right)^{2} \quad R>0 .
$$

Then for some constant $A$ we have,

$$
|\log R(x)| \leq A+1 / \pi \int_{a}^{x} f(t)\left(\psi_{1}(t)+\psi_{2}(t)\right) d t \quad \forall x \in[a, \infty) .
$$

Proof. Consider the modified Prüfer transformation

$$
y=R f^{-1 / 2} \cos \theta, \quad y^{\prime}=-R f^{1 / 2} \sin \theta .
$$

Then we obtain the following differential equations for $R, \theta$ :

$$
\begin{gathered}
\theta^{\prime}=f-(1 / 2) f^{\prime} f^{-1} \sin 2 \theta+g \cos ^{2} \theta+k \cos ^{2} \theta \\
R^{\prime} R^{-1}=(1 / 2) f^{\prime} f^{-1} \cos 2 \theta+(1 / 2) g \sin 2 \theta+(1 / 2) k \sin 2 \theta .
\end{gathered}
$$

We integrate (4.22) over $(a, x)$ in order to obtain the bound in (4.19). The last term of (4.22), due to (4.13), yields a bounded integral. 
Thus we only have to consider the other two terms. The first term in (4.22), using (4.14), yields to

$$
\left|\int_{a}^{x} f^{\prime} f^{-1} \cos 2 \theta d t\right| \leq \int_{a}^{x} \psi_{1} f|\cos 2 \theta| d t .
$$

Now we use (4.21) to obtain,

$$
\begin{aligned}
\left|\int_{a}^{x} f^{\prime} f^{-1} \cos 2 \theta d t\right| \leq & \int_{a}^{x} \psi_{1} \theta^{\prime}|\cos 2 \theta| d t \\
& +\int_{a}^{x} \psi_{1}\left(1 / 2 \psi_{1} f+|g|+|k|\right) d t \\
= & \int_{a}^{x} \psi_{1} \theta^{\prime}(2 / \pi) d t \\
& +\int_{a}^{x} \psi_{1} \theta^{\prime}(|\cos 2 \theta|-2 / \pi) d t \\
& +\int_{a}^{x} \psi_{1}\left(1 / 2 \psi_{1} f+|g|+|k|\right) d t .
\end{aligned}
$$

Substituting $\theta^{\prime}$ from (4.21), we have,

$$
\begin{aligned}
\left|\int_{a}^{x} f^{\prime} f^{-1} \cos 2 \theta d t\right| \leq & \int_{a}^{x} \psi_{1} f(2 / \pi) d t+\int_{a}^{x} \psi_{1} \theta^{\prime}(|\cos 2 \theta|-2 / \pi) d t \\
& +(1+2 / \pi) \int_{a}^{x}\left[(1 / 2) \psi_{1}^{2} f+\psi_{1}|g|+\psi_{1}|k|\right] d t
\end{aligned}
$$

The first integral on the right yields to the term $\psi_{1}$ (see (4.14)). Now we will prove that the other two terms in (4.23) are bounded. Indeed, for the case of the second integral in (4.23), we observe that $\psi_{1}$ is non-negative, non-increasing and that

$$
\int_{a}^{x} \theta^{\prime}(|\cos 2 \theta|-2 / \pi) d t=\int_{a}^{x}(|\cos 2 \theta|-2 / \pi) d t
$$

is uniformly bounded for $x \geq a$. From the mean value theorem for integrals, and since $\int_{a}^{x}\left(\theta^{\prime}|\cos 2 \theta|-2 / \pi\right) d t$ is uniformly bounded, we obtain $\xi \in(a, x)$ such that

$$
\int_{a}^{x} \psi_{1} \theta^{\prime}(|\cos 2 \theta|-2 / \pi) d t=\xi \int_{a}^{x}\left(\theta^{\prime}|\cos 2 \theta|-2 / \pi\right) d t \leq C,
$$

where $C$ is a constant. Hence the second term in (4.23) is bounded. Now in the last integral in (4.23) all three summands of the integrand are in $L^{1}(a, \infty)$. For the first term this was assumed in (4.16). For 
the second term note that $|g| \leq \psi_{2} f$, and again the result follows from (4.16). For the third term note that $\psi_{1}$ is bounded, and then use (4.13). Therefore $\int_{a}^{x}\left|f^{\prime} f^{-1} \cos 2 \theta\right| d t$ is bounded. Similarly we can prove that the second term in (4.22) yields to a bounded integral. (Just replace $\psi_{1}$ by $\psi_{2}$ and $\cos 2 \theta$ by $\sin 2 \theta$.) This concludes the proof of the theorem.

The following theorem is from [2].

Theorem 4.25. Let $r(x)$ be locally $L^{1}(a, \infty)$ and let $\lim _{x \rightarrow \infty} r(x)=$ 0 . Set $p(x)=\sup _{t \geq x}|r(x)|$ and assume that $p \in L^{2}(a, \infty)$. Moreover, let $y$ be a nontrivial solution to $y^{\prime \prime}+(\lambda-r(x)) y=0$, where $x \in[a, \infty)$, and define $R>0$ by $R^{2}=\lambda^{1 / 2} y^{2}+\lambda^{-1 / 2}\left(y^{\prime}\right)^{2}$. Then for fixed $\lambda>0$ and for some fixed constant $A>0$, we have that

$$
|\log R(x)| \leq A+\frac{\lambda^{-1 / 2}}{\pi} \int_{a}^{x} p(x) d t \quad \forall x \in[a, \infty) .
$$

Proof. Apply Theorem 4.17 with $f=\lambda^{1 / 2}, g=-r \lambda^{-1 / 2}, k=0$. The result now is immediate.

Using the above theorem it is possible to prove that the constant $\lambda^{-1 / 2} \pi$ in (4.26) is the best possible, in the way that for any other constant $c<\lambda^{-1 / 2} \pi$ the inequality in (4.26) does not hold.

The Prüfer transformations are extremely useful in obtaining upper bounds for ratios of eigenvalues of certain Differential Operators. Before we close this section we will give a theorem describing the optimal bounds for ratios of eigenvalues of one dimensional Schrödinger Operator with Dirichlet boundary conditions and positive potential.

Theorem 4.27. Let $H=-\frac{d^{2}}{d x^{2}}+V(x)$ be a Schrödinger Operator acting on $L^{2}(I)$, where $I \subset \mathbb{R}$ is a finite closed interval and where Dirichlet boundary conditions are imposed at both endpoints of $I$. Assume that $V \in L^{1}(I)$ and $V(x) \geq 0$ a.e on $I$. Then the ratio $\frac{\lambda_{n}}{\lambda_{1}}$, of the $n^{\text {th }}$ eigenvalue of $H$ to the first eigenvalue of $H$, satisfies the bound $\frac{\lambda_{n}}{\lambda_{1}} \leq n^{2}$. This bound is optimal, and for $V \in L^{2}(I)$ and $n>1$ equality is obtained if and only if $V \equiv 0$ a.e. on $I$.

The proof of the theorem is given in [1]. The Prüfer transformation needed for the proof is

$$
\begin{gathered}
y(x)=r(x) \sin (\sqrt{\lambda} \theta(x)) \\
y^{\prime}(x)=\sqrt{\lambda} r(x) \cos (\sqrt{\lambda} \theta(x)) .
\end{gathered}
$$




\section{REFERENCES}

[1] M.S. Ashbaugh and R. Benguria, Optimal Bounds for ratios of eigenvalues of one dimensional Schrödinger operators with Dirichlet boundary conditions and positive potentials, Comm. of Math. Physics 124 (1989), 403-415.

[2] F.V. Atkinson and W. Everitt, Bound for the point spectrum for a SturmLiouville equation, Proc. of Royal Soc. Edinburgh 80 A (1978), 57-66.

[3] J.H. Barrett and J.S Bradley, Ordinary Differential Equations (1989), 328348 .

[4] G. Birkhoff and G-C. Rota, Ordinary Differential Equations, Blaisdell Publishing Company (1969), 288-308.

[5] R.C. Brown, D.B Hinton and Š. Schwabik, Applications of one-dimensional Sobolev inequality to eigenvalue problems, Differential and Integral Equations 9 (1996), 481-498.

[6] E. Coddington and N. Levinson, Theory of Ordinary Differential Equations, Mc-Graw Hill Book Company (1995), 208-213.

[7] J.H.Cohn, On an oscillation criterion de la Valle Poussin, Quart. Journal of Math. Oxford 39 (1988), 173-174.

[8] W.A. Coppel, Stability and asymptotic Behavior of Differential Equations, D.C. Heath and Company (1965), 123-128.

[9] W.B. Harris, On an Oscillation Criterion Of Cohn, Quart. Journal of Math. Oxford 42 (1991), 309-313.

[10] W.B. Harris and D. Lutz, Asymptotic Integration of adiabatic oscillators, J.Math. Anal. Appl. 51 (1975), 76-93.

[11] P. Hartman On the zeros of solutions of second order linear differential equations, J. London Math. Soc. 27 (1952), 492-496.

[12] P. Hartman, On a class of perturbations of the harmonic oscillator, Proc. AMS 19 (1968), 533-540.

[13] J. Pryce, Numerical solutions of Sturm-Liouville problems, Clarendon Press, Oxford-New York-Tokyo, 1993.

[14] W.T. Reid, Sturmian theory of ordinary differential equations, Mc-Graw Hill Company (1988), 53-62.

[15] M.R. Spiegel, Mathematical Handbook of formulas and tables, McGraw Hill Company, 1968.

George Chailos,

Department of Computer Science,

University of Nicosia,

Nicosia 1700, Cyprus

chailos.g@iunic.ac.cy

Received on 20 August 2008 and in revised version on 28 July 2009. 